\title{
Toward a personalized therapy for panic disorder: preliminary considerations from a work in progress
}

This article was published in the following Dove Press journal:

Neuropsychiatric Disease and Treatment

\author{
Daniela Caldirola ${ }^{1,2}$ \\ Giampaolo Pernal-4 \\ 'Humanitas University, 20090 Pieve \\ Emanuele, Milan, Italy; ${ }^{2}$ Department of \\ Clinical Neurosciences, Hermanas \\ Hospitalarias, Villa San Benedetto Menni \\ Hospital, 22032 Albese Con Cassano, \\ Como, Italy; ${ }^{3}$ Department of Psychiatry \\ and Neuropsychology, Faculty of Health, \\ Medicine and Life Sciences, Maastricht \\ University, 6200 Maastricht, The \\ Netherlands; ${ }^{4}$ Department of Psychiatry \\ and Behavioral Sciences, Leonard Miller \\ School of Medicine, Miami University, \\ Miami, FL 33I36 - I0I5, USA
}

\begin{abstract}
Although several treatment options for panic disorder (PD) are available, the best intervention for each individual patient remains uncertain and the use of a more personalized therapeutic approach in PD is required. In clinical practice, clinicians combine general scientific information and personal experience in the decision-making process to choose a tailored treatment for each patient. In this sense, clinicians already use a somehow personalized medicine strategy. However, the influence of their interpretative personal models may lead to bias related to personal convictions, not sufficiently grounded on scientific evidence. Hence, an effort to give some advice based on the science of personalized medicine could have positive effects on clinicians' decisions. Based on a narrative review of meta-analyses, systematic reviews, and experimental studies, we proposed a first-step attempt of evidence-based personalized therapy for PD. We focused on some phenomenological profiles, encompassing symptoms during/outside panic attacks, related patterns of physiological functions, and some aspects of physical health, which might be worth considering when developing treatment plans for patients with PD. We considered respiratory, cardiac, vestibular, and derealization/depersonalization profiles, with related implications for treatment. Given the extensiveness of the topic, we considered only medications and some somatic interventions. Our proposal should be considered neither exhaustive nor conclusive, as it is meant as a very preliminary step toward a future, robust evidence-based personalized therapy for PD. Clearly much more work is needed to achieve this goal, and recent technological advances, such as wearable devices, big data platforms, and the application of machine learning techniques, may help obtain reliable findings. We believe that combining the efforts of different research groups in this work in progress can lead to largely shared conclusions in the near future.
\end{abstract}

Keywords: pharmacotherapy, somatic, evidence-based, panic

\section{Introduction}

Despite the existence of several therapeutic options for PD, PD treatment continues to pose a challenge because the crucial question about what is the best intervention for each individual patient remains elusive. The current guidelines ${ }^{1-3}$ provide thoughtful recommendations. Pharmacological treatment options for PD include first-line medications [selective serotonin reuptake inhibitors (SSRIs) and serotonin-norepinephrine reuptake inhibitor (SNRI) venlafaxine], second-line medications [tricyclic antidepressants (TCAs), benzodiazepines (BDZs)], and other less supported pharmacological alternatives, whereas cognitive behavioral therapy (CBT) is the first-line psychotherapeutic approach. Some differences
Correspondence: Giampaolo Perna Department of Clinical Neurosciences, Hermanas Hospitalarias, Villa San

Benedetto Menni Hospital, Via Roma 16 22032 Albese con Cassano, Como, Italy

Tel $+3903 \mid 429$ I5II

Fax +39031427246

Email pernagp@gmail.com 
between guidelines exist; however, they mainly share a common view about the mechanism of development of treatment plans for patients with PD: namely, no firstline treatment is recommended as superior to the others, and a combination of treatments is not routinely recommended over monotherapy as initial treatment. Unfortunately, no recommendations considering the unique characteristics of an individual patient, such as symptom profiles, neurobiological functions, biomarkers, or genetic/pharmacogenetics features are available. This gap may partly explain why approximately $20-40 \%$ of the patients in clinical settings do not achieve full remission with the recommended treatments and $25-50 \%$ of the patients relapse within 6 months after discontinuation of drugs, whereas up to $30-50 \%$ still have a full-blown disorder or residual panic-phobic symptoms even after 3-6 years. $^{4,5}$ Hence, further steps toward the use of a more personalized therapeutic approach in PD are required. Clinicians should be provided with additional recommendations, which help them select a treatment with the highest chances of successful outcome for a patient with PD, based on his/her peculiar features. We believe that this strategy may lead to better outcomes in PD treatment, as it has already occurred in other medical fields. ${ }^{6}$ In clinical practice, each clinician behaves in decision making combining general scientific information and personal experience to choose a tailored treatment for each patient. In this sense, clinicians already use a somehow personalized medicine strategy. However, the influence of interpretative personal models may lead to bias related to personal convictions, not sufficiently grounded on scientific evidence. Hence, an effort to give some advice based on the science of personalized medicine could have positive effects on clinicians' decisions.

We are aware that the available evidence-based findings on which to ground a personalized treatment of PD are still limited. Nevertheless, in this proposal, we attempted to present a number of preliminary evidencebased suggestions about how a more personalized treatment plan for a patient with PD can be developed, taking individual clinical symptoms and biological patterns into account. Given the significant comorbidity of PD with medical illnesses and low physical fitness of individuals with PD (Is-PD), ${ }^{7}$ we also evaluated some aspects of the patients' physical health, with the aim of improving both panic symptomatology and physical well-being.

Our proposal should be considered neither exhaustive nor conclusive, as it is meant to be a first, very preliminary step toward a future, robust evidence-based personalized therapy for PD.

\section{Proposal overview}

We based our considerations on a narrative review of meta-analyses, systematic reviews, and experimental studies in the field of PD. Given the extensiveness of the topic, in this first-step proposal, we addressed only a limited number of issues, which might be easily translatable in clinical practice. We focused on some phenomenological profiles, encompassing symptoms during/between panic attacks (PAs), patterns of physiological functions, and some aspects of physical health that might be worth considering when developing treatment plans for Is-PD. We considered only medications and some "somatic" interventions. This choice was not meant to underestimate the multiple psychological aspects that play a significant role in pathophysiology of $\mathrm{PD}$, nor the importance of CBT in the treatment of this disorder. Attentional bias toward somatic sensations, proneness to catastrophic misinterpretation of normal body changes, and interoceptive/exteroceptive conditioning processes are involved in the occurrence of PAs, development of anticipatory anxiety/ maladaptive changes in behavior, and vulnerability to relapse. Likewise, these processes may influence the patterns of physiological functions in Is-PD and somatic symptoms of which they complain. ${ }^{8,9}$ Therefore, we are aware that psychological functioning should be included in phenomenological profiles of Is-PD and considered as target of personalized treatments. Evidence-based recommendations on this topic, through which clinicians can optimize their choices between CBT, medications, or combinations of treatments for each individual with PD, are needed. However, the examination of the complex interplay between these multiple aspects is beyond the aims of this very preliminary, first proposal, and it requires a future, dedicated review.

\section{Phenomenological profiles}

$\mathrm{PD}$ is a heterogeneous psychiatric disorder characterized by recurrent, unexpected PAs, the hallmark of the disorder, persistent concern about additional PAs or their consequences, and/or behavioral modification as a direct result of the PAs; up to $70 \%$ of the Is-PD also have agoraphobia. ${ }^{7}$

According to current nosological systems, a PA in PD must include at least 4 of 13 (DSM5) or 14 (ICD-10) somatic and cognitive symptoms, allowing substantial variability in panic presentation. However, the possibility 
of clinically meaningful panic variants is not considered. Similarly, the possible persistence, outside PAs, of some somatic symptoms with their variants, is not described. Finally, DSM5 acknowledges that at least a portion of IsPD experience induced PAs with administration of sodium lactate infusion/carbon dioxide $\left(\mathrm{CO}_{2}\right)$ inhalation and exhibit hypocapnia and other respiratory irregularities. However, none of these findings are included as valuable features for clinical presentation of PD.

Overall, in contrast to other psychiatric disorders, current nosological systems do not provide any subtypes or specifiers of PAs in PD or of PD. Here, we attempted to define some homogeneous phenomenological profiles of PD, which may be relevant to therapeutic management. We considered respiratory, cardiac, vestibular, and derealization/depersonalization profiles, with related implications for treatment. We are aware that several other phenomenological aspects of panic are worthy of consideration, eg, presentation with nausea or abdominal distress; however, we focused on aspects which are currently more supported by scientific literature. The phenomenological profiles should not be considered as mutually exclusive, but features of different profiles may be simultaneously present to a different extent in a patient with PD.

\section{Respiratory profile Clinical symptoms}

The presence of a subgroup of Is-PD who exhibit prominent respiratory symptoms during PAs received the most consistent endorsement in scientific literature. Historically, Donald Klein proposed the existence of a PD respiratory subtype (RS), with predominant respiratory symptoms, probably linked to a "false suffocation alarm," and an autonomic subtype, with prominent peripheral autonomic symptoms (eg, palpitations). In accordance, empirical evidence of RS and nonrespiratory subtype (NRS) was found, applying cluster and principal components analysis in a large clinical sample. ${ }^{10}$ In the RS, the PAs were characterized by five prominent respiratory symptoms (ie, shortness of breath, choking/smothering sensations, chest pain/discomfort, paresthesias, and fear of dying), along with elevations across most all other panic symptoms, whereas in the NRS, the PAs were typified by low levels of respiratory symptoms, while by high levels of all other panic symptoms. A factor analysis of PA symptoms revealed three dimensions, including a cardiorespiratory dimension with respiratory symptoms and palpitations, ${ }^{11}$ mostly resembling the features of the RS previously found. ${ }^{10}$ Similar dimensions were found in other studies as well. ${ }^{12}$ More recently, latent class and factor mixture analyses of multiple epidemiological samples and a large treatment-seeking sample provided further evidence of the RS and NRS of PAs, with symptoms overlapping those originally found ${ }^{10}$ and high temporal stability. ${ }^{13,14}$ In particular, approximately $65 \%$ of the Is-PD in the clinical sample were typified by a prominent respiratory component, whereas approximately $35 \%$ were characterized by low respiratory symptoms and high general somatic symptoms. ${ }^{13}$ Overall, the RS seemed to represent a more severe form of panic, with a higher familiar prevalence of $\mathrm{PD},{ }^{15}$ increased lifetime psychiatric comorbidity, and increased tendency to seek psychosocial/pharmacological treatments for panic. ${ }^{14}$

Even outside PAs, numerous Is-PD complain of respiratory symptoms during their daily life, and these respiratory complaints are more present in Is-PD than in individuals with generalized anxiety disorder. ${ }^{16}$

\section{Respiratory challenges}

Behavioral hypersensitivity to hypercapnia is a reliable biomarker of vulnerability to PAs, which is present in approximately $50-70 \%$ of the Is-PD and runs in families. Several experimental studies revealed that the RS was associated with a more pronounced hypersensitivity to hypercapnia. Indeed, RS-Is-PD, compared with NRS-IsPD, reported higher rates of induced PAs, with higher respiratory symptoms, during inhalation of hypercapnic gas mixtures (eg, 35\% $\mathrm{CO}_{2} / 65 \% \mathrm{O}_{2}$ ). ${ }^{15,17-19}$ Moreover, RS-Is-PD with the RS exhibited a higher behavioral or respiratory sensitivity to various other laboratory respiratory challenges, such as breath-holding, hyperventilation, or hypoxic test. ${ }^{15}$ Some studies found that Is-PD who panicked with $\mathrm{CO}_{2}$ inhalation/lactate infusion had differences in several respiratory variables during the aforementioned challenges compared with Is-PD who did not panic, such as lower end-tidal $\mathrm{CO}_{2}$ partial pressure $\left(\mathrm{pCO}_{2}\right)$, higher minute ventilation/respiratory rate/tidal volume, prominent symptoms of breathing effort and sense of suffocation, low baseline prechallenge arterial $\mathrm{pCO}_{2}$, and high arterial $\mathrm{pH}^{20}$

\section{Respiratory patterns}

Is-PD have abnormalities in resting-state respiratory patterns compared with healthy controls (HCs) or individuals with generalized anxiety/social anxiety disorder. Is-PD exhibited higher mean minute ventilation, lower end-tidal $\mathrm{pCO}_{2}$, 
higher respiratory variability/irregularity, higher rates of sighs and apneas, when both awake and asleep, reduced $\mathrm{HCO}_{3}{ }^{-} / \mathrm{PO}_{4}{ }^{-}$hematic concentrations (two indexes of chronic hyperventilation), increased respiratory variability during mild physical activity, and impaired diaphragmatic breathing with reduced vital capacity. ${ }^{21-23}$ Significant patterns of instability across numerous autonomic and respiratory variables were found as early as 47 mins before onset of a reallife PA, while the final minutes before PA and the PA onset were characterized by prominent respiratory changes. ${ }^{24}$

Only very scant and inconclusive results are available regarding the association between respiratory patterns and clinical panic presentation. Lower resting end-tidal $\mathrm{pCO}_{2}$ and increased total breath time and variability were found in $\mathrm{RS}$ than in NRS, ${ }^{15}$ whereas no differences in $\mathrm{PO}_{4}{ }^{-}$hematic concentrations between the two subtypes were observed in a small sample of Is-PD. ${ }^{25}$ Specifically in Is-PD, significant correlations were found between resting-state dyspnea during laboratory physiological signals recording and some respiratory variables (ie, tidal volume, minute volume, inspiratory flow rate, and especially end-tidal $\left.\mathrm{pCO}_{2}\right){ }^{26}$

\section{Smoking and respiratory diseases}

Associations between panic, smoking, and asthma have been well established. Cigarette smoking has higher prevalence in Is-PD than in general population or individuals with other anxiety disorders, and an increased risk of panic psychopathology was found among smokers in comparison with nonsmokers. Although smoking and panic have bidirectional influences, growing evidence pointed to the more common pattern of primary smoking and secondary increased likelihood of subsequent PAs/PD. ${ }^{27,28}$ Furthermore, nicotine dependence can contribute to $\mathrm{PD}$ recurrence after remission, ${ }^{29}$ while quitting smoking significantly reduces the risk of both new-onset and persistence of PAs. ${ }^{27}$

Some studies found relationships between smoking and respiratory symptoms/function in Is-PD. Cigarette consumption and behavioral hypersensitivity to hypercapnia were higher in RS-Is-PD than NRS-Is-PD. ${ }^{17}$ Smokers with PD exhibited a significantly higher increase in $35 \% \mathrm{CO}_{2}$ $165 \% \mathrm{O}_{2}$-induced panic symptoms in comparison with nonsmokers with PD. ${ }^{30}$ Smoking worsened the irregularity in resting-state respiratory patterns of Is-PD, and smokers with PD reported more severe spontaneous PAs than nonsmokers without PD. ${ }^{31}$ Overall, these findings support the idea that smoking can facilitate the occurrence of PAs, and at least in a portion of Is-PD, this effect may be related to a peculiar smoking impact on their respiratory function.
Several studies revealed significant associations between $\mathrm{PAs} / \mathrm{PD}$ and asthma/chronic obstructive pulmonary disease (COPD). ${ }^{32-35}$ Asthma was associated with increased crosssectional/longitudinal likelihood of having PAs, with an even greater likelihood in case of severe asthma, and a significant relationship between persistence of asthma and PAs was found as well. ${ }^{32,33}$ Bidirectional longitudinal associations between asthma and PD were reported, even being stronger in smokers compared with nonsmokers. ${ }^{36}$ Moreover, smokers with asthma exhibited higher levels of panic symptoms and increased rates of lifetime PAs/PD than smokers without asthma, ${ }^{37}$ suggesting that the combination of having asthma and being smoker is particularly negative in terms of panic psychopathology. The concurrence of asthma and PD was associated with increased panic symptoms, breathlessness, and hyperventilation during $35 \% \mathrm{CO}_{2} / 65 \% \mathrm{O}_{2}$ challenge, ${ }^{35}$ and with worse asthma outcomes, higher symptom burden, and poorer quality of life. $^{35}$ Eventually, in Is-PD with a current co-diagnosis of asthma, the cardiorespiratory dimension of PAs was significantly higher than other dimensions. ${ }^{11}$

In conclusion, it is plausible that a respiratory profile, with prominent respiratory symptoms and peculiar respiratory sensitivity/patterns, may characterize a more homogeneous part of Is-PD. Furthermore, especially when respiratory symptoms and function are involved, assessing and dealing with smoking habit and respiratory diseases may be of particular relevance. Unfortunately, no study performed comprehensive examination, at the same time and in the same individuals, of all the features whose interplay could typify a respiratory subtype/dimension of Is-PD. Therefore, a complete agreement in terms of the existence and definition of a respiratory subgroup/dimension is still lacking. We believe that in the future, more reliable definition should be based on not only symptoms during PAs, but also it should incorporate symptoms outside PAs, behavioral/respiratory responses to $\mathrm{CO}_{2} /$ respiratory challenges, respiratory function/patterns (recorded both in laboratory and in daily life with wearable devices), smoking habit, and presence of respiratory diseases. However, bearing in mind the current limitations in this field, we proposed below some considerations about treatment of Is-PD with a prominent respiratory profile.

\section{Implications for treatment}

Pharmacological treatment

Studies regarding preferential responses to some medications in Is-PD with a prominent respiratory profile are still 
limited, with mixed results. However, some findings are worth considering. A large placebo-controlled trial found that RS-Is-PD presented better response to imipramine than alprazolam, whereas NRS-Is-PD responded better to alprazolam. ${ }^{10}$ More recently, these preferential responses were not confirmed. ${ }^{14} \mathrm{~A}$ possible explanation of these inconsistencies may arise from differences between imipramine (TCA) and alprazolam (BDZ) in their capability of decreasing hypersensitivity to hypercapnia in Is-PD. Indeed, while consistent results indicated that imipramine decreased hyperreactivity to $\mathrm{CO}_{2}$ after the first days of treatment, $^{38,39}$ the effects of alprazolam are less clear, and the study conducted with the most validated methodology found no significant effects of acute alprazolam premedication on $35 \% \mathrm{CO}_{2}$-induced PAs, when compared to placebo. $^{40}$ Therefore, while alprazolam exhibited a general efficacy for PD treatment clinical trials ${ }^{1}$ and its chronic effects could be different from the acute ones, it may be less effective than other medications in blocking PAs in Is-PD with more prominent respiratory features, namely respiratory symptoms and hyperreactivity to $\mathrm{CO}_{2}$. Conversely, the BDZ clonazepam consistently decreased $35 \% \quad \mathrm{CO}_{2}$-induced PAs after acute and 6-week administration, ${ }^{41}$ and this decrease was associated with short-term successful clinical outcome. ${ }^{41}$ In accordance, RS-Is-PD presented a significantly faster reduction in PAs in the first 8 weeks of treatment with clonazepam than NRS-Is-PD. ${ }^{42}$ Although preliminary, these data suggest that, in case of BDZ use, clonazepam may be preferred in Is-PD with prominent respiratory features.

Several compounds that modulate serotonergic system and have clinical antipanic properties exhibited reliable capability of decreasing $\mathrm{CO}_{2}$-induced PAs in Is-PD. ${ }^{38}$ Imipramine, clomipramine (TCAs), paroxetine, sertraline, and fluvoxamine (SSRIs) decreased behavioral hyperreactivity to $35 \% \mathrm{CO}_{2}$ even after the first week of 30-day treatment, and the decrease of hyperreactivity at the end of treatment was proportional to the degree of clinical symptom improvement. ${ }^{38}$ Similarly, 35\% $\mathrm{CO}_{2}$ hyperreactivity decreased after 1-week treatment with $75 \mathrm{mg}$ of SNRI venlafaxine, a dosage at which venlafaxine acts mainly on the serotonergic system. ${ }^{43}$ Finally, preliminary results showed that successful antipanic treatments with imipramine/clomipramine were associated with normalization of some baseline respiratory parameters, such as hematic $\mathrm{pH}, \mathrm{HCO}_{3}{ }^{-} / \mathrm{PO}_{4}{ }^{-}$, and decreased respiratory responses to hypercapnia. ${ }^{44-46}$ Conversely, reboxetine, a selective noradrenaline reuptake inhibitor, was significantly less effective than paroxetine in decreasing both $35 \% \mathrm{CO}_{2}$-induced PAs and clinical PAs in Is-PD, while the two medications showed similar efficacy in reducing anticipatory anxiety and avoidance. $^{47,48}$ Overall, these findings suggested that, when respiratory function is involved in PD presentation, modulation of noradrenergic system has limited efficacy in reducing PAs. Conversely, modulation of serotonergic system seems to be crucial in reducing PAs, and successful therapeutic outcomes may be related to serotonergic effects on respiratory function in Is-PD with hyperreactivity to hypercapnia and/or respiratory abnormalities. ${ }^{38}$ In line with this, 12-week treatment with paroxetine or escitalopram showed greater improvement in panic symptom severity in RS-Is-PD than in NRS-Is-PD, while the two groups did not differ in general anxiety/ depressive symptom improvement. ${ }^{49}$

Even among serotonergic medications recommended for PD, some compounds might exhibit higher antipanic properties than others in Is-PD with respiratory profile. Preliminary evidence showed that fluvoxamine and imipramine exhibited weaker antipanic properties than sertraline, paroxetine, and clomipramine in Is-PD who were hyperreactive to hypercapnia. ${ }^{38}$ Possible explanations are that fluvoxamine has the lowest in vitro anticholinergic effects, which are thought to be involved in respiratory aspects of antipanic activity, whereas imipramine has the weakest effect on the serotonergic system, which has a crucial role in the panic-respiration connection. ${ }^{38}$ Two-month treatment with paroxetine showed a trend toward a higher percentage of patients without PAs (50\%) than citalopram (24\%), suggesting higher antipanic efficacy of paroxetine, possibly related to its adjunctive anticholinergic properties. ${ }^{50}$ Considering that paroxetine showed direct effects on respiration in animal studies and capability of decreasing respiratory irregularity in Is-PD, ${ }^{51}$ this compound may be particularly suitable for Is-PD with respiratory profile.

Finally, an early decrease in behavioral hyperreactivity to $\mathrm{CO}_{2}$ after the first week significantly predicted good clinical outcome after one-month treatment with SSRIs/ imipramine/clomipramine, ${ }^{38}$ suggesting that $\mathrm{CO}_{2}$ reactivity can be a useful objective predictor of short-term clinical outcome in Is-PD with respiratory profile.

Thus, considering some subjective and objective respiratory measures in Is-PD might be helpful for treatment. In Is-PD with features of the respiratory profile, 
paroxetine and sertraline among SSRIs, clomipramine among TCAs, and clonazepam among BDZ might be more suitable for reducing PAs, whereas noradrenergic compounds have weaker efficacy (Box 1 and Table 1).

\section{Somatic treatment}

Several studies evaluated the clinical efficacy of breathing therapies (BRTs) in PD, suggesting the usefulness of including this intervention in treatment plans, especially in Is-PD with prominent respiratory profile. A 4-week capnometry-assisted BRT, which was aimed to increase end-tidal $\mathrm{pCO}_{2}$ and reduce respiration rate (RR), provided significant improvement of panic symptoms and increase of $\mathrm{pCO}_{2}$ from hypocapnia to normocapnia at the endpoint and up to 12-month follow-up. ${ }^{52,53}$ Treatment success was not related to respiratory symptom subtype or initial level of $\mathrm{pCO}_{2}$. However, the majority of patients were hypocapnic at the beginning of the treatment, and patients who continued to show abnormal levels of $\mathrm{pCO}_{2}$ or RR during recovery from a voluntary hyperventilation test were less likely to have clinical improvement, ${ }^{52}$ suggesting that BRT should be particularly recommended to Is-PD with respiratory features. Similarly, a comparison between capnometry-assisted BRT and cognitive training suggested that both

Box I Proposed assessment to define phenomenological profiles

\begin{tabular}{|l|}
\hline Pretreatment assessment \\
\hline Measurement of panic symptoms during/outside PAs through specific \\
symptomatological scales. \\
Evaluation of smoking habit, physical activity level, asthma, COPD, \\
CDs. \\
Recording of resting-state respiratory patterns; $35 \% \mathrm{CO}_{2} / 65 \% \mathrm{O}_{2}$ \\
challenge test. \\
Recording of cardiac and autonomic patterns (ECG/Holter monitor/ \\
HRV measurement). \\
Cardiopulmonary exercise testing. \\
Blood sampling: levels of PO ${ }_{4}^{-}, \mathrm{HCO}_{3}{ }^{-}$; CRP/inflammatory markers; \\
PDW, MPV, RDW; total/LDL/HDL cholesterol. \\
In addition, if prominent vestibular symptoms and/or severe Ag and/ \\
or Ag not remitted with recommended treatments: \\
Otoneurological examinations/posturography \\
In addition, if prominent D/DSs during/outside PAs: \\
EEG \\
Otoneurological examinations/posturography
\end{tabular}

Abbreviations: $\mathrm{Ag}$, agoraphobia; $\mathrm{CDs}$, cardiac diseases; $\mathrm{CO}_{2}$, carbon dioxide; COPD, chronic obstructive pulmonary disease; CRP, C-reactive protein; D/DSs, derealization/depersonalization symptoms; ECG, electrocardiogram; EEG, electroencephalogram; $\mathrm{HCO}_{3}{ }^{-}$, bicarbonate; $\mathrm{HDL}$, high-density lipoprotein; HRV, heart rate variability; LDL, low-density lipoprotein; MPV, mean platelet volume; $\mathrm{O}_{2}$, oxygen; PAs, panic attacks; PDW, platelet distribution width; $\mathrm{PO}_{4}^{-}$, phosphate; RDW, red cell distribution width. interventions could provide clinical benefits in PD, while only BRT led to corrections from initially hypocapnia to normocapnia; furthermore, in $\mathrm{BRT}, \mathrm{pCO}_{2}$ unidirectionally preceded and mediated improvement of symptom appraisal, perceived control, and panic symptoms. ${ }^{54}$ This suggested that, although panic improvement can be achieved through different pathways, BRT might be particularly relevant for Is-PD with hypocapnia. In line with this, changes in $\mathrm{pCO}_{2}$ during capnometry-assisted BRT mediated changes in anxiety sensitivity (AS) of Is-PD, but not vice versa, ${ }^{55}$ and low baseline $\mathrm{pCO}_{2}$ predicted poorer outcome from behavioral treatment. ${ }^{56}$ These preliminary findings suggest that to involve BRT in treatment plans can provide additional benefit to reduce AS and improve efficacy of CBT in Is-PD with respiratory profile. It should be noted that both opposite BRTs, aimed to decrease or increase end-tidal $\mathrm{pCO}_{2}$, reduced panic severity in a sample of Is-PD, ${ }^{57}$ suggesting that clinical improvement might depend on factors common to BRTs, such as restoring regular breathing. Finally, BRT aimed to restore impaired diaphragmatic breathing ameliorated previously reduced percent vital capacity of Is-PD, ${ }^{23}$ supporting the idea that BRT can directly improve abnormalities in respiratory function of Is-PD.

Finally, BRT might ameliorate clinical control of asthma, panic symptoms, and quality of life in individuals with comorbid PD and asthma, and although controlled trials are needed, adapting CBT protocols specifically for comorbid PD and asthma/COPD is thought to provide benefits to clinical outcomes. ${ }^{58,59}$ Based on the findings discussed earlier, smoking prevention and quitting smoking programs should become one of the relevant steps in PD treatment, particularly when respiratory function is involved in PD presentation.

In conclusion, a careful assessment of pretreatment characteristics, including respiratory symptoms during/ outside PAs, objective physiological measurements of respiratory functioning, smoking habit, respiratory diseases, and behavioral/respiratory responses to respiratory challenges, might help to develop a treatment plan more suitable for Is-PD with respiratory profile (Box 1 and Table 1). It should be noted that end-tidal $\mathrm{pCO}_{2}$, respiratory patterns, and $\mathrm{HCO}_{3}{ }^{-} / \mathrm{PO}_{4}{ }^{-}$hematic concentrations can be relatively simply measured; thus, their use might be easily translatable in clinical practice. The reassessment of both subjective and objective measures over the course of the treatment might help clinicians monitor treatment efficacy. 
Table I Proposed implications for evidence-based treatment in phenomenological profiles of PD

\begin{tabular}{|c|c|}
\hline Phenomenological profile ${ }^{a}$ & Proposed implications for treatment \\
\hline \multicolumn{2}{|l|}{ Respiratory profile } \\
\hline $\begin{array}{l}\text { Prominent RSs during/outside PAs. } \\
\uparrow \text { Behavioral/respiratory sensitivity to } 35 \% \mathrm{CO}_{2} / 65 \% \mathrm{O}_{2} \text { chal- } \\
\text { lenge. } \\
\text { Abnormalities in respiratory patterns: } \downarrow \text { end-tidal } \mathrm{PCO}_{2} \text {, } \\
\text { respiratory irregularity, } \downarrow \mathrm{HCO}_{3}{ }^{-} / \mathrm{PO}_{4}{ }^{-} \text {hematic levels. } \\
\text { Presence of current smoking. } \\
\text { Presence of current/lifetime asthma/COPD. }\end{array}$ & $\begin{array}{l}\text { Pharamacological treatments to reduce PAs: among SSRIs, paroxetine/sertaline; } \\
\text { among TCAs, clomipramine; among BDZs, clonazepam. } \\
\text { Noradrenergic compounds not recommended. } \\
\text { Somatic treatments to reduce PAs: BRT, aerobic exercise, quitting smoking } \\
\text { programs. }\end{array}$ \\
\hline \multicolumn{2}{|l|}{ Cardiac profile } \\
\hline $\begin{array}{l}\text { Prominent CSs during/outside PAs. } \\
\text { Abnormalities in cardiac/autonomic patterns: } \downarrow \text { HRV, impaired } \\
\text { circardian BP pattern, ECG abnormalities. } \\
\text { Low cardiopulmonary fitness, exercise avoidance. } \\
\text { Presence of current/lifetime CDs. } \\
\text { Presence of current smoking. } \\
\text { Presence of additional CVRFs: } \uparrow C R P, \uparrow P D W, \uparrow M P V, \uparrow R D W \text {, } \\
\uparrow \text { cholesterol, } \uparrow \text { homocysteine. }\end{array}$ & $\begin{array}{l}\text { No data about preferential clinical responses to medications. } \\
\text { Medications with more favorable profile on cardiac function: paroxetine, sertraline } \\
\text { (with regular monitoring of cholesterol levels). } \\
\text { TCAs not recommended. } \\
\text { Clonazepam: caution in Is-PD with } \downarrow \text { HRV. } \\
\text { Somatic treatments: aerobic exercise; healthy dietary patterns (Mediterranean } \\
\text { diet, omega 3-fatty acids/probiotics), quitting smoking programs, BRT. }\end{array}$ \\
\hline \multicolumn{2}{|l|}{ Vestibular profile } \\
\hline $\begin{array}{l}\text { Prominent VSs during/outside PAs. } \\
\text { Abnormalities in balance system function: impaired postural } \\
\text { control, } \uparrow \text { sensitivity to visual-vestibular patterns. }\end{array}$ & $\begin{array}{l}\text { Pharamacological treatments: citalopram, sertraline, imipramine (clinical observa- } \\
\text { tions). } \\
\text { Somatic treatments: vestibular rehabilitation. }\end{array}$ \\
\hline \multicolumn{2}{|l|}{ Derealization/Depersonalization profile } \\
\hline $\begin{array}{l}\text { Prominent D/DSs during/outside PAs } \\
\text { EEG abnormalities. }\end{array}$ & $\begin{array}{l}\text { No data about preferential clinical responses to medications. } \\
\text { In case of EEG abnormalities: evaluate the opportunity of using clonazepam/ } \\
\text { anticonvulsants. }\end{array}$ \\
\hline
\end{tabular}

Note: ${ }^{a}$ The higher the number of features, the higher the prominence of the phenomenological profile.

Abbreviations: BDZs, benzodiazepines; BP, blood pressure; BRT, breathing therapy; CDs, cardiac diseases; $\mathrm{CO}_{2}$, carbon dioxide; $\mathrm{COPD}$, chronic obstructive pulmonary disease; CRP, C-reactive protein; CVRFs, cardiovascular risk factors; CSs, cardiac symptoms; D/DSs, derealization/depersonalization symptoms; ECG, electrocardiogram; $\mathrm{EEG}$, electroencephalogram; $\mathrm{HCO}_{3}{ }^{-}$, bicarbonate; $\mathrm{HRV}$, heart rate variability; Is-PD, individuals suffering from panic disorder; MPV, mean platelet volume; $\mathrm{O}_{2}$, oxygen; $\mathrm{PAs}$, panic attacks; $\mathrm{PCO}_{2}$, partial pressure $\mathrm{CO}_{2}$; $\mathrm{PD}$, panic disorder; $\mathrm{PDW}$, platelet distribution width; $\mathrm{PO}_{4}{ }^{-}$, phosphate; $\mathrm{RDW}$, red cell distribution width; $\mathrm{RSs}$, respiratory symptoms; SNRIs, serotonin-norepinephrine reuptake inhibitor; SSRIs, selective serotonin reuptake inhibitors; TCAs, tricyclic antidepressants; VSs, vestibular symptoms; $\uparrow$, high; $\downarrow$, low.

\section{Cardiac profile Clinical symptoms}

Palpitations, pounding heart/accelerated heart rate (HR) (cardiac symptoms, CSs), are among the most frequent symptoms that Is-PD experience during spontaneous/ induced PAs and outside PAs during daily life. ${ }^{60}$ Cluster analyses did not find a qualitatively distinct "autonomic/ cardiac" subtype, while CSs were present in both RS and NRS, along with other general somatic symptoms. ${ }^{10,13}$ From a dimensional point of view, CSs were significantly associated with respiratory symptoms in PA presentation. ${ }^{11}$ Some studies using ambulatory monitoring found objective increase of HR during real-life PAs; similarly, increased HR and systolic/diastolic blood pressure during $\mathrm{CO}_{2}$-induced
PAs were found. ${ }^{60}$ In clinical practice, it is well known that CSs are particularly alarming for Is-PD. Is-PD who experience intense CSs are often scared of dying from a heart attack during PAs, and they typically undergo cardiac examinations to receive medical reassurances.

\section{Cardiac patterns}

Experimental findings revealed subclinical abnormalities of autonomic and cardiac function in Is-PD when compared to HCs. ${ }^{7,61}$ Most studies found imbalanced autonomic regulation, reduced heart rate variability (HRV) (lower parasympathetic activity and higher sympathetic/parasympathetic ratio), both at rest and during mental stress, impaired circadian blood pressure pattern with inadequate nighttime 
reduction of blood pressure, as well as instability of HR during sleep. Significant autonomic instability was found as early as 47 mins before the onset of a real-life PA, with marked increase of HR the minute before PA onset. ${ }^{24}$ A defective neuronal noradrenaline reuptake in the heart of Is-PD was also found, which might augment the sympathetic cardiac firing. Surface electrocardiograms showed that Is-PD have increased variability in the QT interval, which augmented even more during hyperventilation, and increased dispersion of the QT and $P$-wave. These indexes reflect higher ventricular repolarization time variability, and higher regional heterogeneity of ventricular repolarization and atrial depolarization, respectively. Further, Is-PD exhibited higher variability of electrocardiographic (ECG) R-wave amplitude after beta-adrenergic stimulation with isoproterenol.

Is-PD exhibited exercise avoidance in their daily life, ${ }^{62,63}$ and they displayed poorer cardiovascular fitness than HCs, as suggested by the findings of lower levels of maximal oxygen consumption and decreased exercise tolerance during cardiopulmonary exercise testing. ${ }^{63-65}$ Interestingly, somatic, rather than cognitive, symptoms of anxiety predict lower levels of physical activity in Is-PD, suggesting that exercise avoidance in these patients may be partly depending on the perception of their low global physical fitness. ${ }^{7,62}$ Finally, in naturalistic conditions, and outside PAs, a preliminary ambulatory monitoring study found reduced correlations between $\mathrm{HR}$ and physical activity in Is-PD, indicating reduced efficiency of metabolic coupling than HCs. ${ }^{66}$

Overall, the cardiovascular system of Is-PD seemed to present several subclinical abnormalities and to be somehow less efficient compared with HCs.

\section{Cardiac diseases and cardiovascular risk factors}

The association between PD and cardiac diseases (CDs) is well established. ${ }^{61,67}$ Several cardiovascular risk factors (CVRFs), which can play a role in this association, were found in Is-PD. CVRFs directly related to cardiac function were described earlier and involved decreased HRV, impaired autonomic regulation of HR/blood pressure, subclinical abnormalities of cardiac repolarization/ depolarization, and low cardiovascular fitness. Additional CVRFs in Is-PD were also found, when compared to $\mathrm{HCs}$, including increased arterial stiffness and poorer endothelial function, as indicated by elevated plasma homocysteine levels, lower nitric oxide levels, and increased platelet aggregation. ${ }^{68,69}$ Furthermore, previous studies in Is-PD found increased platelet distribution width and mean platelet volume, two hematological markers of platelet activation, and increased red cell distribution width, a measure associated with inflammation and cardiovascular diseases. ${ }^{70,71}$ Several studies showed IsPD to exhibit higher levels of hematological markers of inflammation (proinflammatory cytokines, C-reactive protein) than $\mathrm{HCs}$, and preliminary results found impaired oxidative balance and higher oxidative stress. $^{72-74}$ Finally, unhealthy lifestyle associated with $\mathrm{PD}$, such as exercise avoidance and smoking habit, can be additional CVRFs.

In summary, due to the relevance of CSs in panic presentation, the involvement of autonomic/cardiac system in pathophysiology of $\mathrm{PD}$, and the associations between PD, CVRFs, and CDs, the cardiac aspects are worthy of being assessed and considered in treatment of PD, as we proposed below.

\section{Implications for treatment}

Pharmacological treatment

Studies evaluating preferential clinical responses to some medications in Is-PD with prominent CSs and/or subclinical cardiac abnormalities are lacking. However, as several medications used in PD influence the cardiac function, some experimental findings might be considered, when choosing/monitoring treatments in Is-PD. Imipramine and nortriptyline were found to reduce $\mathrm{HRV}$ in Is-PD, while paroxetine increased HRV and decreased the relative cardiac sympathetic activity in these patients. ${ }^{75-77}$ Similarly, an increased variability in the QT interval was found in Is-PD treated with nortriptyline, whereas it was not found in those treated with paroxetine. ${ }^{78}$ Thus, paroxetine may be a medication of choice particularly in Is-PD with reduced HRV and/or subclinical abnormalities of cardiac repolarization, whereas TCAs should be used more cautiously. However, these possible favorable effects of paroxetine on cardiac function should be confirmed because the available studies in Is-PD are scant, with small sample sizes, and one of them found the unfavorable effect of reducing cardiac vagal function. ${ }^{76}$ Preliminary findings showed that Is-PD responders to 6/12-week pharmacotherapy with mixed antidepressants exhibited improvement in HRV, suggesting that changes in autonomic regulation might help in monitoring the course of treatments in PD. ${ }^{79,80}$ Concerning BZDs, only one study examined the effect of 4-week clonazepam treatment on 
HRV of Is-PD presenting with palpitations. ${ }^{81}$ Compared with placebo, clonazepam led to a decrease in all the time and frequency domain measures of HRV, in line with other studies that found reduction of HRV and vagal tone following BDZ administration in humans. ${ }^{82}$ Although these very preliminary results need confirmation, they suggest caution when using clonazepam in IsPD who present pretreatment low HRV. Conversely, preliminary clinical observations suggested that clonazepam might provide benefits in the treatment of atrial fibrillation in some selected Is-PD; ${ }^{83}$ however, clinical controlled trials are lacking.

TCAs seemed to prolong the QT interval to a greater extent than SSRIs. As a group, SSRIs were associated with small but statistically significant effect on QT, with citalopram and escitalopram related to the greatest QT prolongation, while paroxetine and fluoxetine did not exhibit significant effects on QT length compared to placebo. However, the overall magnitude of SSRIs-related QT prolongation seemed to have very limited clinical significance in the vast majority of patients, particularly in those with no other risk factors for torsades de pointes, ${ }^{84,85}$ while more attention should be paid to patients taking multiple medications due to possible additive effects on QT length. When using venlafaxine, the possibility of mild dosedependent increase in HR and blood pressure should be considered. $^{85}$

Finally, associations were found between SSRIs treatment and increased levels of total/high-density lipoprotein/low-density lipoprotein cholesterol in Is-PD, especially with paroxetine and sertraline. ${ }^{86,87}$ Thus, evaluation of pretreatment cholesterol levels and a careful monitoring over the course of SSRIs therapy are recommended.

Other effects of SSRIs may be beneficial in Is-PD presenting with CVRFs. Sertraline exhibited capability of improving endothelial function and reducing inflammatory markers in patients with coronary heart diseases (CHDs), and it elicited coronary vasodilatation in in vitro experiments. Paroxetine, sertraline, and citalopram inhibited platelet aggregation/activation in patients with CHDs, and paroxetine increased nitric oxide plasma levels in HCs and depressed patients. ${ }^{88}$ Although no data on Is-PD are available, it is conceivable that these properties of SSRIs can be protective against cardiovascular morbidity in Is-PD, particularly in those who present markers of inflammation and/or impaired endothelial function.
In conclusion, in Is-PD presenting abnormalities in cardiac patterns or CVRFs, paroxetine and sertraline may present a more favorable profile, even though cholesterol level assessments are needed, while TCAs are not recommended (Box 1 and Table 1).

\section{Somatic treatment}

Aerobic exercise seemed to be effective as an adjunctive intervention for Is-PD. Indeed, although exercise alone was less effective compared with paroxetine/clomipramine or $\mathrm{CBT}^{89}$ it provided additional benefits in reducing panic/ anxiety symptoms when combined with standard treatments. Eight/twelve weeks of moderate/intense aerobic exercise on regular basis added clinical benefits to medications or CBT. ${ }^{90,91}$ Preliminary results showed accelerating therapeutic effects of 30-min moderate/intense aerobic exercise prior to exposure sessions within an exposure-based CBT, ${ }^{92}$ while a 12-day high-intensity interval training provided additional clinical improvement in medicated Is-PD. ${ }^{93}$ Aerobic exercise on a regular basis also improved the reduced maximal oxygen consumption during cardiopulmonary exercise testing and endurance performance in Is-PD, ${ }^{91,93}$ while acute moderate/intense exercise before $35 \% \mathrm{CO}_{2}$ challenge significantly reduced $\mathrm{CO}_{2}$ panicogenic effects. ${ }^{94}$ These findings suggested that exercise has direct favorable effects on the impaired cardiorespiratory function of these patients. Thus, practicing aerobic exercise on regular basis should be recommended to Is-PD, especially to those with CSs/autonomic imbalance/CVRFs, but also to Is-PD with the respiratory profile, due to the well-known link between cardiac function and respiration. ${ }^{95}$ Furthermore, it is conceivable that BRT may provide some benefits on cardiac symptoms and function of Is-PD with "cardiac profile," considering the respiratory-cardiac connection, and the above-mentioned worsening in cardiac parameters during hyperventilation in Is-PD; however, studies directly exploring this topic are lacking. Finally, beyond physical exercise, other cardiovascular risk reduction strategies should be recommended in Is-PD with "cardiac profile," including smoking cessation and healthy dietary patterns, such as Mediterranean diet and nutrient supplements with omega 3-fatty acids/probiotics, which can contribute to reduce inflammation, oxidative stress, and cholesterol levels. $^{73,96}$

In conclusion, pretreatment assessment of cardiac symptom/function and CVRFs is recommended when developing treatment plan for Is-PD (Box 1 and Table 1). It should be noted that ECG and hematological parameters associated with cardiovascular risk can be simply assessed 
in clinical routine, while cardiopulmonary fitness and HRV measurements might be translatable in clinical practice in a relatively easy way.

\section{Vestibular profile Clinical symptoms}

Dizziness, lightheadedness, and feeling like fainting (vestibular symptoms, VSs) are present in approximately 50 $-70 \%$ of the Is-PD, both during and outside PAs. ${ }^{12,60}$ A distinct vestibular subtype was not found with cluster analyses of PA symptoms, while VSs were present across both the RS and NRS, along with other general somatic symptoms. ${ }^{10,13}$ From a dimensional point of view, VSs were associated to sweating, trembling, nausea, and chills/hot flashes in a "mixed somatic" dimension of PA presentation. ${ }^{11}$ Preliminary findings indicated a significant relationship between the presence of dizziness during PAs and agoraphobia, as well as observations in clinical practice suggest an association between dizziness outside PAs during daily life and presence/severity of agoraphobia. ${ }^{97}$

\section{Vestibular patterns}

Several studies explored balance system function in Is-PD through standardized otoneurologic examinations. When compared to HCs, Is-PD who complained of dizziness, and Is-PD not selected for the presence of VSs, exhibited higher rate (up to $50-70 \%$ ) of vestibular abnormalities, which generally did not represent full-blown vestibular disorders. Overall, studies in Is-PD consistently found that subclinical abnormalities in balance system function, especially postural instability and impaired visual-vestibular interactions, were significantly associated with presence and severity of agoraphobia, and with complaints of VSs, ${ }^{12,97-101}$ in line with observations in clinical practice. Overall, individual sensitivity to visuo-vestibular unusual patterns and its impact in postural control might contribute to panic and agoraphobia at least in a portion of Is-PD. ${ }^{102}$ Finally, a single study found association between symptomatological reactivity to $35 \% \mathrm{CO}_{2}$ challenge and vestibular abnormalities in Is-PD. ${ }^{97}$ This is in line with the wellknown interplay between respiratory and vestibular systems, based on which hyperventilation can induce VSs and, vice versa, VSs can trigger respiratory discomfort. ${ }^{97}$

\section{Implications for treatment}

Pharmacological treatment

Studies exploring responses to medications in Is-PD with VSs and/or subclinical vestibular abnormalities are scant, and no comparative studies are available. A prospective case series including 9 Is-PD with chronic dizziness found improvement in panic symptoms, dizziness, and some otoneurologic parameters, after 3-month treatment with imipramine. These favorable effects of imipramine, which has serotonergic properties, were thought to be related to the well-known involvement of serotonergic pathways in both panic and balance system regulation. ${ }^{99,103}$ In line with this, in a mixed sample of patients with dizziness, vestibular abnormalities, and psychiatric disorders, including a large portion of Is-PD, SSRIs relieved dizziness to a greater extent than vestibular suppressants or BDZ. ${ }^{104}$ In an open study, we found that 6-week citalopram treatment improved static posturography abnormalities and panic-phobic symptoms in a small sample of Is-PD. Interestingly, the two patients whose abnormal posturography lasted to the end point were still agoraphobic, while the patients whose posturography improved were no longer agoraphobic by the end of the trial, ${ }^{99}$ supporting the idea that balance system abnormalities might contribute to maintenance of agoraphobia. Finally, in our clinical experience, Is-PD who have agoraphobia and dizziness achieve greater improvement with sertraline than with other SSRIs. Although this idea needs confirmation, we speculate that, for Is-PD with vestibular profile, serotonergic compounds with adjunctive effects on histaminergic (citalopram) or dopaminergic (sertraline) properties might be particularly suitable because both these systems are involved in balance modulation, along with the serotonergic system (Box 1 and Table 1). ${ }^{4}$

\section{Somatic treatment}

Findings on this topic are very limited. Only one open study found that a small sample of Is-PD with agoraphobia, dizziness, and vestibular abnormalities, who reported limited improvement after a brief (4 weeks) CBT program, obtained further significant benefits in panic-phobic symptoms and dizziness with 8/12-week vestibular rehabilitation. ${ }^{105}$ Similarly, unpublished data of our group indicated that in a small group of Is-PD with agoraphobia, dizziness and vestibular abnormalities, who were partial responders to SSRIs combined with CBT, 10 sessions of vestibular rehabilitation with peripheral visual stimulation obtained significant improvement of panic-phobic symptoms, dizziness, and posturographic parameters. Although these findings are very preliminary and need confirmation, they suggest that vestibular rehabilitation might be considered in Is-PD with "vestibular profile," as an adjunctive 
intervention to recommended treatments. Finally, it is conceivable that to introduce in CBT programs some interventions/exposures specifically targeted on dizziness/dizziness-eliciting situations might be particularly useful in these patients.

In conclusion, although connections between panic-phobic symptoms and balance system were consistently found, the possible existence of treatments more suitable for Is-PD with "vestibular profile" received limited consideration. Nevertheless, based on the available knowledge, otoneurologic examinations are recommended in Is-PD with VSs and agoraphobia, especially in case of poor responses to standard treatments. When developing treatment plans for Is-PD with vestibular profile, the very preliminary observations about citalopram, sertraline, imipramine, and vestibular rehabilitation might be considered (Box 1 and Table 1).

\section{Derealization/depersonalization profile}

We would like to mention briefly the issue of Is-PD with derealization and/or depersonalization symptoms (D/DSs), which received limited attention in the scientific literature. No distinct D/D subtype was found with cluster analyses, while derealization was associated with fear of going crazy and fear of losing control in a "cognitive" dimension of PA presentation. ${ }^{11}$ Approximately $24-40 \%$ of the Is-PD seemed to present D/DSs during PAs, while up to 50 $-60 \%$ experienced these symptoms outside PAs. While the findings about relation between D/DSs and childhood trauma in Is-PD are inconclusive, the presence of D/DSs was associated with greater clinical severity, higher psychiatric comorbidity, poorer response to treatments, and electroencephalographic (EEG) abnormalities, especially in the temporal regions. ${ }^{4}$ To the best of our knowledge, no studies evaluated preferential responses to different treatments in Is-PD with D/DSs. However, some preliminary findings suggested that anticonvulsant medications, which, except clonazepam, are not approved for use in $\mathrm{PD}$, provide clinical benefits in patients with $\mathrm{PD}$ having EEG abnormalities. ${ }^{4}$ Based on this limited evidence, careful screening of D/DSs and possibly associated EEG abnormalities are worthy of consideration in clinical practice. Future clinical studies are needed to select appropriate treatments and evaluate the potential effectiveness of anticonvulsants in patients with PD with this peculiar profile. Finally, one study found an association between D/DSs and VSs in a subgroup of Is-PD. ${ }^{106}$ Considering that patients with vestibular disorders often complain of derealization as a symptom associated with dizziness or vertigo, otoneurologic examinations in Is-PD with D/DSs might be recommended to evaluate the possible presence of vestibular abnormalities (Box 1 and Table 1).

\section{Conclusions}

Our first-step proposal of personalized therapy for PD has several limitations. Mainly, we focused on a limited part of the aspects involved in the pathophysiology and course of PD, and we considered only medications and some "somatic" treatments. Clearly, a future, more comprehensive proposal should be developed, which takes into account multiple factors, such as each person's psychological features and within-person relationships between different variables, and which considers CBT and combination of treatments.

In conclusion, much more work is needed to achieve an evidence-based personalized therapy for PD. Recent technological advance might help identify reliable profiles of Is-PD and define treatments tailored on specific features of each patient, such as wearable devices recording real-time physiological signals, symptoms, perceptions, and behaviors; the storage of clinical/research data in shared "big data" platforms; the application of machine learning techniques on these data to achieve reliable findings. We hope that combining the efforts of different research groups in this work in progress can lead to largely shared conclusions in the near future.

\section{Acknowledgments}

The authors would like to thank Alice Riva, MSc, and Erika Sangiorgio, PsyD, for their assistance with the preparation of the manuscript. The authors would also like to thank Enago for the English language review.

\section{Disclosure}

The authors report no conflicts of interest in this work.

\section{References}

1. American Psychiatric Association. Practice guideline for the treatment of patients with panic disorder. 2009. Available from: https://psychia tryonline.org/pb/assets/raw/sitewide/practice_guidelines/guidelines/ panicdisorder.pdf. Accessed June 9, 2018.

2. National Institute for Health and Clinical Excellence. Generalised anxiety disorder and panic disorder (with or without agoraphobia) in adults, management. 2011. Available from: https://www.nice.org.uk/ guidance/cg113. Accessed June 9, 2018.

3. Bandelow B, Michaelis S, Wedekind D. Treatment of anxiety disorders. Dialogues Clin Neurosci. 2017;19(2):93-107.

4. Caldirola D, Alciati A, Riva A, Perna G. Are there advances in pharmacotherapy for panic disorder? A systematic review of the past five years. Expert Opin Pharmacother. 2018;19(12):1357-1368. doi:10.1080/14656566.2018.1504921 
5. Perna G, Caldirola D. Management of treatment-resistant panic disorder. Curr Treat Options Psychiatry. 2017;4(4):371-386. doi:10.1007/s40501-017-0128-7

6. Perna G, Grassi M, Caldirola D, Nemeroff CB. The revolution of personalized psychiatry: will technology make it happen sooner? Psychol Med. 2018;48(5):705-713. doi:10.1017/S0033291717002859

7. Perna $G$, Caldirola D. Is panic disorder a disorder of physical fitness? A heuristic proposal. F1000Res. 2018;7:294. doi:10.12688/f1000research

8. Bouton ME, Mineka S, Barlow DH. A modern learning theory perspective on the etiology of panic disorder. Psychol Rev. 2001;108(1):4-32.

9. De Cort K, Griez E, Buchler M, Schruers K. The role of "interoceptive" fear conditioning in the development of panic disorder. Behav Ther. 2012;43(1):203-215. doi:10.1016/j.beth.2011.06.005

10. Briggs AC, Stretch DD, Brandon S. Subtyping of panic disorder by symptom profile. Br J Psychiatry. 1993;163:201-209.

11. Meuret AE, White KS, Ritz T, Roth WT, Hofmann SG, Brown TA. Panic attack symptom dimensions and their relationship to illness characteristics in panic disorder. J Psychiatr Res. 2006;40 (6):520-527. doi:10.1016/j.jpsychires.2005.09.006

12. Kircanski K, Craske MG, Epstein AM, Wittchen HU. Subtypes of panic attacks: a critical review of the empirical literature. Depress Anxiety. 2009;26(10):878-887. doi:10.1002/da.20603

13. Roberson-Nay R, Kendler KS. Panic disorder and its subtypes: a comprehensive analysis of panic symptom heterogeneity using epidemiological and treatment seeking samples. Psychol Med. 2011;41(11):2411-2421. doi:10.1017/S0033291711000547

14. Roberson-Nay R, Latendresse SJ, Kendler KS. A latent class approach to the external validation of respiratory and non-respiratory panic subtypes. Psychol Med. 2012;42 (3):461-474. doi:10.1017/S0033291711001425

15. Freire RC, Perna G, Nardi AE. Panic disorder respiratory subtype: psychopathology, laboratory challenge tests, and response to treatment. Harv Rev Psychiatry. 2010;18(4):220-229. doi:10.3109/10673229.2010.493744

16. Wilhelm FH, Trabert W, Roth WT. Physiologic instability in panic disorder and generalized anxiety disorder. Biol Psychiatry. 2001;49 (7):596-605

17. Biber B, Alkin T. Panic disorder subtypes: differential responses to CO2 challenge. Am J Psychiatry. 1999;156(5):739-744. doi:10.1176/ajp.156.5.739

18. Valenca AM, Nardi AE, Nascimento I, Zin WA, Versiani M. Respiratory panic disorder subtype and sensitivity to the carbon dioxide challenge test. Braz J Med Biol Res. 2002;35(7):783-788. doi:10.1590/s0100-879x2002000700004

19. Zugliani MM, Freire RC, Perna G, Crippa JA, Nardi AE. Laboratory, clinical and therapeutic features of respiratory panic disorder subtype. CNS Neurol Disord Drug Targets. 2015;14(5):627-635.

20. Niccolai V, van Duinen MA, Griez EJ. Respiratory patterns in panic disorder reviewed: a focus on biological challenge tests. Acta Psychiatr Scand. 2009;120(3):167-177. doi:10.1111/j.16000447.2009.01408.x

21. Grassi M, Caldirola D, Di Chiaro NV, et al. Are respiratory abnormalities specific for panic disorder? A meta-analysis. Neuropsychobiology. 2014;70(1):52-60. doi:10.1159/000364830

22. Grassi M, Caldirola D, Vanni G, et al. Baseline respiratory parameters in panic disorder: a meta-analysis. J Affect Disord. 2013;146 (2):158-173. doi:10.1016/j.jad.2012.08.034

23. Yamada T, Inoue A, Mafune K, Hiro H, Nagata S. Recovery of percent vital capacity by breathing training in patients with panic disorder and impaired diaphragmatic breathing. Behav Modif. 2017;41(5):665-682. doi:10.1177/0145445517711436

24. Meuret AE, Rosenfield D, Wilhelm FH, et al. Do unexpected panic attacks occur spontaneously? Biol Psychiatry. 2011;70 (10):985-991. doi:10.1016/j.biopsych.2011.05.027
25. Beria P, Viana ACW, Behenck A, et al. Respiratory subtype of panic disorder: can serum phosphate levels be a possible outcome to group cognitive-behavior therapy? $J$ Affect Disord. 2018;235:474-479. doi:10.1016/j.jad.2018.04.002

26. Burkhardt SC, Wilhelm FH, Meuret AE, Blechert J, Roth WT. Temporal stability and coherence of anxiety, dyspnea, and physiological variables in panic disorder. Biol Psychol. 2010;85 (2):226-232. doi:10.1016/j.biopsycho.2010.07.005

27. Bakhshaie J, Zvolensky MJ, Goodwin RD. Cigarette smoking and the onset and persistence of panic attacks during mid-adulthood in the United States: 1994-2005. J Clin Psychiatry. 2016;77(1):e21e24. doi:10.4088/JCP.14m09290

28. Cosci F, Knuts IJ, Abrams K, Griez EJ, Schruers KR. Cigarette smoking and panic: a critical review of the literature. J Clin Psychiatry. 2010;71(5):606-615. doi:10.4088/JCP.08r04523blu

29. Liu Y, Sareen J, Bolton J, Wang J. Development and validation of a risk-prediction algorithm for the recurrence of panic disorder. Depress Anxiety. 2015;32(5):341-348. doi:10.1002/da.22359

30. Knuts IJ, Cosci F, Esquivel G, et al. Cigarette smoking and 35\% $\mathrm{CO}(2)$ induced panic in panic disorder patients. J Affect Disord. 2010;124(1-2):215-218. doi:10.1016/j.jad.2009.10.012

31. Caldirola D, Bellodi L, Cammino S, Perna G. Smoking and respiratory irregularity in panic disorder. Biol Psychiatry. 2004;56 (6):393-398. doi:10.1016/j.biopsych.2004.06.013

32. Goodwin RD, Eaton WW. Asthma and the risk of panic attacks among adults in the community. Psychol Med. 2003;33 (5):879-885.

33. Goodwin RD, Pine DS, Hoven CW. Asthma and panic attacks among youth in the community. J Asthma. 2003;40(2):139-145.

34. Perna G, Bertani A, Politi E, Colombo G, Bellodi L. Asthma and panic attacks. Biol Psychiatry. 1997;42(7):625-630. doi:10.1016/ S0006-3223(96)00436-2

35. Boudreau M, Bacon SL, Paine NJ, et al. Impact of panic attacks on bronchoconstriction and subjective distress in asthma patients with and without panic disorder. Psychosom Med. 2017;79(5):576-584. doi:10.1097/PSY.0000000000000443

36. Hasler G, Gergen PJ, Kleinbaum DG, et al. Asthma and panic in young adults: a 20-year prospective community study. Am J Respir Crit Care Med. 2005;171(11):1224-1230. doi:10.1164/rccm. 200412-1669OC

37. Johnson AL, McLeish AC. Differences in panic psychopathology between smokers with and without asthma. Psychol Health Med. 2017;22(1):110-120. doi:10.1080/13548506.2016.1153676

38. Perna G, Bertani A, Caldirola D, Gabriele A, Cocchi S, Bellodi L. Antipanic drug modulation of $35 \% \mathrm{CO} 2$ hyperreactivity and short-term treatment outcome. J Clin Psychopharmacol. 2002;22 (3):300-308.

39. Sardinha A, Freire RC, Zin WA, Nardi AE. Respiratory manifestations of panic disorder: causes, consequences and therapeutic implications. J Bras Pneumol. 2009;35(7):698-708. doi:10.1590/ s1806-37132009000700012

40. Pols H, Verburg K, Hauzer R, Meijer J, Griez E. Alprazolam premedication and $35 \%$ carbon dioxide vulnerability in panic patients. Biol Psychiatry. 1996;40(9):913-917. doi:10.1016/00063223(95)00494-7

41. Valenca AM, Nardi AE, Nascimento I, et al. Early carbon dioxide challenge test may predict clinical response in panic disorder. Psychiatry Res. 2002;112(3):269-272.

42. Nardi AE, Valenca AM, Nascimento I, et al. A three-year follow-up study of patients with the respiratory subtype of panic disorder after treatment with clonazepam. Psychiatry Res. 2005;137(1-2):61-70. doi:10.1016/j.psychres.2005.05.011

43. Bertani A, Bellodi L, Bussi R, Caldirola D, Cucchi M, Perna G. The effect of one-week treatment with venlafaxine on $35 \% \mathrm{CO} 2$ hyperreactivity in patients with panic disorder: an open study. J Clin Psychopharmacol. 2003;23(1):106-108. 
44. Gorman JM, Browne ST, Papp LA, et al. Effect of antipanic treatment on response to carbon dioxide. Biol Psychiatry. 1997;42 (11):982-991.

45. Gorman JM, Fyer AJ, Ross DC, et al. Normalization of venous $\mathrm{pH}$, pCO2, and bicarbonate levels after blockade of panic attacks. Psychiatry Res. 1985;14(1):57-65.

46. Perez-Costillas L, Montes MR, Martinez-Ortega JM, et al. Phosphate levels as a possible state marker in panic disorder: preliminary study of a feasible laboratory measure for routine clinical practice. J Psychiatr Res. 2013;47(10):1357-1362. doi:10.1016/j.jpsychires.2013.05.022

47. Perna G, Bertani A, Caldirola D, et al. Modulation of hyperreactivity to $35 \% \mathrm{CO} 2$ after one week of treatment with paroxetine and reboxetine: a double-blind, randomized study. $J$ Clin Psychopharmacol. 2004;24(3):277-282.

48. Bertani A, Perna G, Migliarese G, et al. Comparison of the treatment with paroxetine and reboxetine in panic disorder: a randomized, single-blind study. Pharmacopsychiatry. 2004;37 (5):206-210. doi:10.1055/s-2004-832593

49. Song HM, Kim JH, Heo JY, Yu BH. Clinical characteristics of the respiratory subtype in panic disorder patients. Psychiatry Investig. 2014;11(4):412-418. doi:10.4306/pi.2014.11.4.412

50. Perna G, Bertani A, Caldirola D, Smeraldi E, Bellodi L. A comparison of citalopram and paroxetine in the treatment of panic disorder: a randomized, single-blind study. Pharmacopsychiatry. 2001;34(3):85-90. doi:10.1055/s-2001-14283

51. Yeragani VK, Rao R, Tancer M, Uhde T. Paroxetine decreases respiratory irregularity of linear and nonlinear measures of respiration in patients with panic disorder. A preliminary report. Neuropsychobiology. 2004;49(2):53-57. doi:10.1159/ 000076410

52. Meuret AE, Wilhelm FH, Ritz T, Roth WT. Feedback of end-tidal pCO2 as a therapeutic approach for panic disorder. J Psychiatr Res. 2008;42(7):560-568. doi:10.1016/j.jpsychires.2007.06.005

53. Tolin DF, McGrath PB, Hale LR, Weiner DN, Gueorguieva R. A multisite benchmarking trial of capnometry guided respiratory intervention for panic disorder in naturalistic treatment settings. Appl Psychophysiol Biofeedback. 2017;42(1):51-58. doi:10.1007/ s10484-017-9354-4

54. Meuret AE, Rosenfield D, Seidel A, Bhaskara L, Hofmann SG. Respiratory and cognitive mediators of treatment change in panic disorder: evidence for intervention specificity. J Consult Clin Psychol. 2010;78(5):691-704. doi:10.1037/a0019552

55. Meuret AE, Rosenfield D, Hofmann SG, Suvak MK, Roth WT. Changes in respiration mediate changes in fear of bodily sensations in panic disorder. $J$ Psychiatr Res. 2009;43(6):634-641. doi:10.1016/j.jpsychires.2008.08.003

56. Davies CD, Craske MG. Low baseline $\mathrm{pCO} 2$ predicts poorer outcome from behavioral treatment: evidence from a mixed anxiety disorders sample. Psychiatry Res. 2014;219(2):311-315. doi:10.1016/j.psychres.2014.06.003

57. Kim S, Wollburg E, Roth WT. Opposing breathing therapies for panic disorder: a randomized controlled trial of lowering vs raising end-tidal $\mathrm{P}(\mathrm{CO} \square) . \quad J \quad$ Clin Psychiatry. 2012;73(7):931-939. doi: $10.4088 /$ JCP. $11 \mathrm{~m} 07068$

58. Livermore N, Sharpe L, McKenzie D. Panic attacks and panic disorder in chronic obstructive pulmonary disease: a cognitive behavioral perspective. Respir Med. 2010;104(9):1246-1253. doi:10.1016/j.rmed.2010.04.011

59. Laurino RA, Barnabe V, Saraiva-Romanholo BM, et al. Respiratory rehabilitation: a physiotherapy approach to the control of asthma symptoms and anxiety. Clinics (Sao Paulo). 2012;67 (11):1291-1297. doi:10.6061/clinics/2012(11)12

60. Leibold NK, Schruers KR. Assessing panic: bridging the gap between fundamental mechanisms and daily life experience. Front Neurosci. 2018;12:785. doi:10.3389/fnins.2018.00044
61. Caldirola D, Schruers KR, Nardi AE, et al. Is there cardiac risk in panic disorder? An updated systematic review. J Affect Disord. 2016;194:38-49. doi:10.1016/j.jad.2016.01.003

62. Belem Da Silva CT, Schuch F, Costa M, Hirakata V, Manfro GG. Somatic, but not cognitive, symptoms of anxiety predict lower levels of physical activity in panic disorder patients. $J$ Affect Disord. 2014;164:63-68. doi:10.1016/j.jad.2014.04.007

63. Muotri RW, Bernik MA. Panic disorder and exercise avoidance. Rev Bras Psiquiatr. 2014;36(1):68-75. doi:10.1590/1516-44462012-1012

64. Caldirola D, Namia C, Micieli W, et al. Cardiorespiratory response to physical exercise and psychological variables in panic disorder. Rev Bras Psiquiatr. 2011;33(4):385-389.

65. Muotri RW, Bernik MA, Neto FL. Misinterpretation of the Borg's Rating of Perceived Exertion Scale by patients with panic disorder during ergospirometry challenge. BMJ Open Sport Exerc Med. 2017;3(1):e000164. doi:10.1136/bmjsem-2017-000232

66. Pfaltz MC, Kolodyazhniy V, Blechert J, et al. Metabolic decoupling in daily life in patients with panic disorder and agoraphobia. $J$ Psychiatr Res. 2015;68:377-383. doi:10.1016/j.jpsychires. 2015.04.027

67. Machado S, Sancassiani F, Paes F, et al. Panic disorder and cardiovascular diseases: an overview. Int Rev Psychiatry. 2017;29 (5):436-444. doi:10.1080/09540261.2017.1357540

68. Meier C, Harbrecht U, Liedtke R, et al. Relative hyperhomocysteinemia in patients with panic disorder: a case-control study. Neuropsychobiology. 2010;62(3):164-170. doi:10.1159/000318573

69. Yapislar H, Aydogan S, Ozum U. Biological understanding of the cardiovascular risk associated with major depression and panic disorder is important. Int J Psychiatry Clin Pract. 2012;16 (1):27-32. doi:10.3109/13651501.2011.620127

70. Asoglu M, Aslan M, Imre O, et al. Mean platelet volume and red cell distribution width levels in initial evaluation of panic disorder. Neuropsychiatr Dis Treat. 2016;12:2435-2438. doi:10.2147/NDT. $\mathrm{S} 111108$

71. Kokacya MH, Copoglu US, Kivrak Y, et al. Increased mean platelet volume in patients with panic disorder. Neuropsychiatr Dis Treat. 2015;11:2629-2633. doi:10.2147/NDT.S94147

72. Glaus J, von Kanel R, Lasserre AM, et al. The bidirectional relationship between anxiety disorders and circulating levels of inflammatory markers: results from a large longitudinal population-based study. Depress Anxiety. 2018;35(4):360-371. doi:10.1002/da.22710

73. Perna G, Iannone G, Alciati A, Caldirola D. Are anxiety disorders associated with accelerated aging? A focus on neuroprogression. Neural Plast. 2016;2016:8457612. doi:10.1155/2016/5260671

74. Quagliato LA, Nardi AE. Cytokine alterations in panic disorder: A systematic review. J Affect Disord. 2018;228:91-96. doi:10.1016/j.jad.2017.11.094

75. Tucker P, Adamson P, Miranda R Jr, et al. Paroxetine increases heart rate variability in panic disorder. J Clin Psychopharmacol. 1997;17(5):370-376.

76. Yeragani VK, Jampala VC, Sobelewski E, Kay J, Igel G. Effects of paroxetine on heart period variability in patients with panic disorder: a study of holter ECG records. Neuropsychobiology. 1999;40 (3):124-128. doi:10.1159/000026608

77. Yeragani VK, Rao R. Effect of nortriptyline and paroxetine on measures of chaos of heart rate time series in patients with panic disorder. J Psychosom Res. 2003;55(6):507-513.

78. Yeragani VK, Pohl R, Jampala VC, et al. Effects of nortriptyline and paroxetine on QT variability in patients with panic disorder. Depress Anxiety. 2000;11(3):126-130.

79. Choi KW, Jang EH, Kim AY, et al. Heart rate variability for treatment response between patients with major depressive disorder versus panic disorder: A 12-week follow-up study. J Affect Disord. 2019;246:157-165. doi:10.1016/j.jad.2018.12.048 
80. Prasko J, Latalova K, Diveky T, et al. Panic disorder, autonomic nervous system and dissociation - changes during therapy. Neuro Endocrinol Lett. 2011;32(5):641-651.

81. Baker B, Khaykin Y, Devins G, et al. Correlates of therapeutic response in panic disorder presenting with palpitations: heart rate variability, sleep, and placebo effect. Can J Psychiatry. 2003;48 (6):381-387. doi:10.1177/070674370304800604

82. Chalmers JA, Quintana DS, Abbott MJ, Kemp AH. Anxiety disorders are associated with reduced heart rate variability: a meta-analysis. Front Psychiatry. 2014;5:80. doi:10.3389/ fpsyt.2014.00080

83. Kahn JP, Veras AB, Nardi AE, Curtis AB. Clonazepam treatment of atrial fibrillation in panic anxiety patients: a case series. Psychosomatics. 2018. doi:10.1016/j.psym.2018.12.001

84. Beach SR, Kostis WJ, Celano CM, et al. Meta-analysis of selective serotonin reuptake inhibitor-associated QTc prolongation. J Clin Psychiatry. 2014;75(5):e441-e449. doi:10.4088/JCP.13r08672

85. Celano CM, Daunis DJ, Lokko HN, Campbell KA, Huffman JC. Anxiety disorders and cardiovascular disease. Curr Psychiatry Rep. 2016;18(11):101. doi:10.1007/s11920-016-0739-5

86. Herran A, Ramirez ML, Carrera M, et al. Panic disorder, treatment with selective serotonin reuptake inhibitors, and cholesterol levels. J Clin Psychopharmacol. 2006;26(5):538-540. doi:10.1097/01. jcp.0000237941.56107.b7

87. Le Melledo JM, Mailo K, Lara N, et al. Paroxetine-induced increase in LDL cholesterol levels. J Psychopharmacol. 2009;23 (7):826-830. doi:10.1177/0269881108094320

88. Nezafati MH, Eshraghi A, Vojdanparast M, Abtahi S, Nezafati P. Selective serotonin reuptake inhibitors and cardiovascular events: A systematic review. J Res Med Sci. 2016;21:66. doi:10.4103/ 1735-1995.189647

89. Bartley CA, Hay M, Bloch MH. Meta-analysis: aerobic exercise for the treatment of anxiety disorders. Prog Neuropsychopharmacol Biol Psychiatry. 2013;45:34-39. doi:10.1016/j.pnpbp.2013.04.016

90. Gaudlitz K, Plag J, Dimeo F, Strohle A. Aerobic exercise training facilitates the effectiveness of cognitive behavioral therapy in panic disorder. Depress Anxiety. 2015;32(3):221-228. doi:10.1002/ da. 22337

91. Lamego MK, Lattari E, Souza de Sá Filho A, et al. Aerobic exercise reduces anxiety symptoms and improves fitness in patients with panic disorder. Med Express (São Paulo, Online). 2016;3(3): M160306.

92. Bischoff S, Wieder G, Einsle F, et al. Running for extinction? Aerobic exercise as an augmentation of exposure therapy in panic disorder with agoraphobia. J Psychiatr Res. 2018;101:34-41. doi:10.1016/j.jpsychires.2018.03.001
93. Plag J, Ergec DL, Fydrich T, Strohle A. High-intensity interval training in panic disorder patients: a pilot study. J Nerv Ment Dis. 2019;207(3):184-187. doi:10.1097/NMD.0000000000000944

94. Esquivel G, Diaz-Galvis J, Schruers K, et al. Acute exercise reduces the effects of a $35 \% \mathrm{CO} 2$ challenge in patients with panic disorder. $J$ Affect Disord. 2008;107(1-3):217-220. doi:10.1016/j.jad.2007.07.022

95. Elstad M, O’Callaghan EL, Smith AJ, Ben-Tal A, Ramchandra R. Cardiorespiratory interactions in humans and animals: rhythms for life. Am J Physiol Heart Circ Physiol. 2018;315(1):H6-H17. doi:10.1152/ajpheart.00701.2017

96. Morkl S, Wagner-Skacel J, Lahousen T. et al. The role of nutrition and the gut-brain axis in psychiatry: a review of the literature. Neuropsychobiology. 2018;1-9. doi:10.1159/000492834

97. Perna G, Dario A, Caldirola D, et al. Panic disorder: the role of the balance system. J Psychiatr Res. 2001;35(5):279-286.

98. Caldirola D, Teggi R, Bondi S, et al. Is there a hypersensitive visual alarm system in panic disorder? Psychiatry Res. 2011;187 (3):387-391. doi:10.1016/j.psychres.2010.05.012

99. Perna G, Alpini D, Caldirola D, Raponi G, Cesarani A, Bellodi L. Serotonergic modulation of the balance system in panic disorder: an open study. Depress Anxiety. 2003;17(2):101-106. doi:10.1002/ da. 10092

100. Teggi R, Caldirola D, Bondi S, Perna G, Bellodi L, Bussi M. Vestibular testing in patients with panic disorder and chronic dizziness. Acta Otorhinolaryngol Ital. 2007;27(5):243-247.

101. Teggi R, Caldirola D, Colombo B, et al. Dizziness, migrainous vertigo and psychiatric disorders. J Laryngol Otol. 2010;124 (3):285-290. doi:10.1017/S0022215109991976

102. Coelho CM, Balaban CD. Visuo-vestibular contributions to anxiety and fear. Neurosci Biobehav Rev. 2015;48:148-159. doi:10.1016/j. neubiorev.2014.10.023

103. Mezzasalma MA, Mathias Kde V, Nascimento I, Valenca AM, Nardi AE. Imipramine for vestibular dysfunction in panic disorder: a prospective case series. Arq Neuropsiquiatr. 2011;69 (2A):196-201

104. Staab JP, Ruckenstein MJ, Solomon D, Shepard NT. Serotonin reuptake inhibitors for dizziness with psychiatric symptoms. Arch Otolaryngol Head Neck Surg. 2002;128(5):554-560.

105. Jacob RG, Whitney SL, Detweiler-Shostak G, Furman JM. Vestibular rehabilitation for patients with agoraphobia and vestibular dysfunction: a pilot study. J Anxiety Disord. 2001;15 (1-2):131-146.

106. Cox BJ, Swinson RP. Instrument to assess depersonaliza tion-derealization in panic disorder. Depress Anxiety. 2002;15 (4):172-175. doi:10.1002/da.10051
Neuropsychiatric Disease and Treatment

\section{Publish your work in this journal}

Neuropsychiatric Disease and Treatment is an international, peerreviewed journal of clinical therapeutics and pharmacology focusing on concise rapid reporting of clinical or pre-clinical studies on a range of neuropsychiatric and neurological disorders. This journal is indexed on PubMed Central, the 'PsycINFO' database and CAS, and is the official journal of The International Neuropsychiatric Association (INA). The manuscript management system is completely online and includes a very quick and fair peer-review system, which is all easy to use. Visit http://www.dovepress.com/testimonials.php to read real quotes from published authors. 